\title{
The Solutions of Initial Value Problems for Second-order Integro-differential Equations with Delayed Arguments in Banach Spaces
}

\author{
Tingting Guan* \\ School of Mathematics and Computer Science, Shanxi Normal University, Linfen, Shanxi 041004, P. R. China \\ *Corresponding author: guantingting1985@163.com
}

Received June 24, 2015; Revised November 26, 2015; Accepted December 04, 2015

\begin{abstract}
By using the partial order method and some new comparison results, the maximal or minimal solution of the initial value problem for nonlinear second order integro-differential equations with delayed arguments in Banach spaces are investigated. In this paper, we require only a lower solution or an upper solution and some weaker conditions presented here, and we extend and improve some recent results (see [1-11]).
\end{abstract}

Keywords: second-order integro-differential equation, delayed arguments, measure of non-compactness, solution, monotone iterative technique

Cite This Article: Tingting Guan, "The Solutions of Initial Value Problems for Second-order Integrodifferential Equations with Delayed Arguments in Banach Spaces.” Turkish Journal of Analysis and Number Theory, vol. 3, no. 6 (2015): 154-159. doi: 10.12691/tjant-3-6-3.

\section{Introduction}

The theory of differential equations with deviated argument is very important and significant branch of nonlinear analysis. It is worthwhile mentioning that differential equations with deviated argument appear often in investigations connected with mathematical physics, mechanics, engineering, economics and so on (cf. $[10,11,12]$, for example). One of the basic problems considered in the theory of differential equations with deviated argument is to establish convenient conditions guaranteeing the existence of solutions of those equations, we refer to some recent papers [13,14,15,16,17] and references.

Let $E$ be a real Banach space with $\|\cdot\|$ and let $P$ be a cone in $E$. The partial order " $\leq$ " is introduced by cone $P$, i.e., $x, y \in E, x \leq y$ if and only if $y-x \in P$. A cone $P$ is said to be normal if there exist a constant $N_{P}>0$ such that $x, y \in E, \quad \theta \leq x \leq y$ implies $\|x\| \leq N_{P}\|y\| ; N_{P}$ is called the normal constant of $P$. Recall that a cone $P$ is said to be regular if every increasing and bounded in order sequence in $E$ has a limit, i.e., $x_{1} \leq x_{2} \leq \ldots \leq x_{n} \leq \ldots \leq y$ implies $\left\|x_{n}-x\right\| \rightarrow 0$ as $n \rightarrow \infty$ for some $x \in E$. The regularity of $P$ implies the normality of $P$. Let $E^{*}$ be the dual space of $E, P^{*}=\left\{\varphi \in E^{*} \mid \varphi(x) \geq 0, \forall x \in P\right\}$ is called the dual cone. Obviously, $x \in P$ if and only if $\varphi(x) \geq 0$, for all $\varphi \in P^{*}$. Let $\quad P_{C}=\{u \in C[J, E]: u(t) \geq \theta$ for all $t \in J\}$, where $J=[0, a](a>0)$ and $C[J, E]$ denotes the Banach space of all continuous mapping $u: J \rightarrow E$ with the norm
$\|u\|_{C}=\max \{\|u(t)\|: u \in J\}$. It is clear that $P_{C}$ is a cone of the $C[J, E]$ and so it defines a partial ordering in $C[J, E]$. Obviously, the normality of $P$ implies the normality of $P_{C}$ and the normal constants of $P_{C}$ and $P$ are the same. For further details on cone theory, one can refer to $[3,8,9]$. Let

$$
\begin{aligned}
& C^{1}[J, E]=\{u: J \rightarrow E \mid u(t) \text { continuously differentiable }\}, \\
& C^{2}[J, E] \\
& =\left\{\begin{array}{r}
u: J \rightarrow E \mid u(t) \text { second } \\
\quad \text { order continuously differentiable }
\end{array}\right\} .
\end{aligned}
$$

In this paper, we consider the solutions for the following initial value problems (IVP) of nonlinear second-order integro-differential equations of mixed type in ordered Banach spaces $E$,

$$
\left\{\begin{array}{l}
u^{\prime \prime}(t)=f\left(\begin{array}{l}
t, u(t), u(\beta(t)), \\
u^{\prime}(t), T u(t), S u(t)
\end{array}\right) \equiv F u(t), \\
u(0)=x_{0}, u^{\prime}(0)=x_{1},
\end{array}\right.
$$

where $\quad t \in J, \quad x_{0}, x_{1} \in E, \quad \beta \in C[J, J]$, $F \in C[J \times E \times E \times E \times E \times E, E]$, and

$$
\begin{gathered}
\left(T_{u}\right)(t)=\int_{0}^{t} k(t, s) u(s) d s,\left(S_{u}\right)(t)=\int_{0}^{a} h(t, s) u(s) d s . \\
k(t, s) \in C\left[D, R^{+}\right], h(t, s) \in C\left[D_{0}, R^{+}\right], \\
D=\left\{(t, s) \in R^{2} \mid 0 \leq s \leq t \leq a\right\},
\end{gathered}
$$




$$
D_{0}=\left\{(t, s) \in R^{2} \mid(t, s) \in J \times J\right\}, R^{+}=[0,+\infty) .
$$

Let

$$
\begin{aligned}
& k_{0}=\max \{k(t, s) \mid(t, s) \in D\}, \\
& h_{0}=\max \left\{h(t, s) \mid(t, s) \in D_{0}\right\} .
\end{aligned}
$$

For any $B \subset C[J, E], t \in J$, let

$$
\begin{aligned}
& B(t)=\{u(t) \mid u \in B\}, T B(t)=\{(T u)(t) \mid u \in B\}, \\
& S B(t)=\{(S u)(t) \mid u \in B\} .
\end{aligned}
$$

The solutions for initial value problems (IVP) of nonlinear first-order integro-differential equations of mixed type in ordered Banach spaces have made considerable headway in recent years (see $[2,6]$ ). But there has been little discussion for the solutions of (IVP) (1.1). In the special case where $f$ does not contain $u(\beta(t))$ and $u^{\prime}(t)$, the solutions for initial value problems (IVP) (1.1) in Banach spaces have some results (see [1,5]). In another special case where $f$ does not contain $u(\beta(t))$, in [4], Su obtained some new results by using Mönch fixed point theorem and new comparison results.

In this paper, we first establish a new comparison theorem, and then, by requiring only a lower solution or an upper solution and some weaker conditions, we investigate the existence of the minimal or maximal solutions of the (IVP) (1.1), where $f$ contains $u^{\prime}, S u$ and delayed arguments $u(\beta(t))$ under the conditions which are more extensive than those in $[1,5]$.

\section{Several Lemmas}

The following comparison results and lemmas play an important role in this paper.

Lemma 1. (Comparison theorem) Assume that $E$ is a Banach space, $P$ is a cone in $E, \beta(t) \leq t$ on $J$, and $u=u(t) \in C^{2}[J, E]$ satisfies

$$
\left\{\begin{array}{l}
u^{\prime \prime}(t) \geq-M u(t)-K u(\beta(t))-N u^{\prime}(t)-L(T u)(t) \\
u(0)=\theta, u^{\prime}(0) \geq \theta
\end{array}\right.
$$

where $M, K, N, L$ are non-negative constants, and provided one of the following two conditions hold

(i) $\left[3(M+K) a+6 N+L k_{0} a^{2}\right] a \leq 6$,

(ii) $\quad N>0, \quad L k_{0}\left(2-e^{N a}\right)+(M+K) N\left(e^{N a}-1\right)$ $+L k_{0} N\left(a e^{N a}+a-e^{N a}\right)-(M+K) N a \leq N^{3}$.

Then $u(t) \geq \theta, u^{\prime}(t) \geq \theta, \forall t \in J$.

Proof. For any $\varphi \in P^{*}$, let $p(t)=\varphi(u(t)), \forall t \in J$. then

$$
\begin{aligned}
& p(\beta(t))=\phi(u(\beta(t))), p^{\prime \prime}(t)=\varphi\left(u^{\prime \prime}(t)\right), \\
& p^{\prime}(t)=\varphi\left(u^{\prime}(t)\right),(T p)(t)=\varphi((T u)(t)), \forall t \in J .
\end{aligned}
$$

Thus, by (2.1) we have that $\left\{\begin{array}{l}p^{\prime \prime}(t) \geq-M p(t)-K p(\beta(t))-N p^{\prime}(t)-L(T p)(t), \forall \in J, \\ p(0)=0, p^{\prime}(0) \geq 0 .\end{array}\right.$

Let $\quad p_{1}(t)=p^{\prime}(t)$, then $\quad p_{1}(t) \in C^{1}[J, R], \quad$ and $p(t)=\int_{0}^{t} p_{1}(s) d s$. Hence, we have that

$$
\left\{\begin{aligned}
p_{1}^{\prime}(t) \geq & -\int_{0}^{t}\left[M+L \int_{0}^{t} k(t, r) d r\right] p_{1}(s) d s \\
& -K \int_{0}^{\beta(t)} p_{1}(s) d s-N p_{1}(t), \forall t \in J, \\
p_{1}(0) \geq & 0 .
\end{aligned}\right.
$$

Now, we shall prove that $p_{1}(t) \geq 0, \forall t \in J$.

In the case of condition (i), if $p_{1}(t) \geq 0$ is not true , then there is a to $t_{0} \in(0, a]$ such that $p_{1}\left(t_{0}\right)<0$. Let $\max \left\{p_{1}(t): 0 \leq t \leq t_{0}\right\}=\lambda$, then $\lambda \geq 0$.

If $\lambda=0$, then $p_{1}(t) \leq 0, \forall t \in\left[0, t_{0}\right]$. Then, by (2.2), we have $p_{1}^{\prime}(t) \geq 0, \forall t \in\left[0, t_{0}\right]$. So, $p_{1}(t)$ is increasing in $\left[0, t_{0}\right]$, we have $p_{1}\left(t_{0}\right) \geq p_{1}(0) \geq 0$, which contradicts $p_{1}\left(t_{0}\right)<0$.

If $\lambda>0$, then there exists a $t_{1} \in\left[0, t_{0}\right]$ such that $p_{1}\left(t_{1}\right)=\lambda>0$. From (2.2), we have

$$
\begin{aligned}
p_{1}^{\prime}(t) & \geq-\lambda \int_{0}^{t}\left[M+L k_{0}(t-s)\right] d s-\lambda K \beta(t)-\lambda N \\
& =-\lambda\left(M t+K t+\frac{L k_{0} t^{2}}{2}+N\right), \forall t \in\left[0, t_{0}\right] .
\end{aligned}
$$

Thus, we have that

$$
\begin{aligned}
p_{1}\left(t_{0}\right) & =p_{1}\left(t_{1}\right)+\int_{t_{1}}^{t_{0}} p_{1}^{\prime}(s) d s \\
& \geq \lambda-\lambda \int_{0}^{a}\left(M s+K s+\frac{L k_{0} s^{2}}{2}+N\right) d s \\
& =\lambda\left(1-\frac{(M+K) a^{2}}{2}-\frac{L k_{0} a^{3}}{6}-N a\right)
\end{aligned}
$$

Then, by $p_{1}\left(t_{0}\right)<0$, we have $\left[\begin{array}{c}3(M+K) a \\ +6 N+L k_{0} a^{2}\end{array}\right] a>6$, which contradicts $(i)$.

In the case of condition (ii) holding, let

$$
\omega(t)=p_{1}(t) e^{N t}
$$

and applying it to (2.2), by a similar process, we can obtain $\omega(t) \geq 0, \forall t \in J$, and so $p_{1}(t) \geq 0, \forall t \in J$.

Therefore, $\quad p^{\prime}(t) \geq 0, \quad \forall t \in J$, which implies that $p(t) \geq p(0)=0, \quad \forall t \in J$. By the arbitrarily of $\varphi \in P^{*}$, we have $u(t) \geq \theta, u^{\prime}(t) \geq \theta, \quad \forall t \in J$.

Lemma 1 is proved. 
Lemma 2. [3] Let $B \subset C[J, E]$ be countable and bounded, then $\alpha(B(t)) \in L\left[J, R^{+}\right]$, and

$$
\alpha\left(\left\{\int_{J} u(t) d t: u \in B\right\}\right) \leq 2 \int_{J} \alpha(B(t)) d t
$$

Lemma 3. [3] Let $B \subset C[J, E]$ be countable and equicontinuous, let $m(t)=\alpha(B(t)), \forall t \in J$, then $m(t)$ is continuous on $J$ and

$$
\alpha\left(\left\{\int_{J} B(s) d s\right\}\right) \leq \int_{J} \alpha(B(s)) d s .
$$

Lemma 4. $[2,6]$ Assume that $m \in C\left[J, R^{+}\right]$satisfies $m(t) \leq M_{1} \int_{0}^{t} m(s) d s+M_{2} t \int_{0}^{t} m(s) d s+M_{3} t \int_{0}^{a} m(s) d s, \forall t \in J$. where $M_{1}>0, \quad M_{2} \geq 0, \quad M_{3} \geq 0$ are constants. Then $m(t) \equiv 0, \forall t \in J$, provided one of the following two conditions holds

(i) $a M_{3}\left(e^{a\left(M_{1}+a M_{2}\right)}-1\right)<M_{1}+a M_{2}$,

(ii) $a\left(2 M_{1}+a M_{2}+a M_{3}\right)<2$.

\section{Main Results}

We list for convenience the following assumptions.

$\left(H_{1}\right)$ : (i) There exists $u_{0} \in C^{2}[J, E]$ satisfying

$$
u_{0}^{\prime \prime}(t) \leq F u_{0}(t), t \in J, u_{0}(0)=x_{0}, u_{0}^{\prime}(0) \leq x_{1} .
$$

(ii) There exists $v_{0} \in C^{2}[J, E]$ satisfying

$$
v_{0}^{\prime \prime} \geq F v_{0}(t), t \in J, v_{0}(0)=x_{0}, v_{0}^{\prime}(0) \geq x_{1} .
$$

$\left(H_{2}\right)$ : (i) Whenever $t \in J$ and $u_{i}, v_{i}(i=1,2) \in G$ $\equiv\left\{\omega \in C^{1}[J, E] \mid \omega \geq u_{0}, \omega^{\prime} \geq u_{0}^{\prime}\right\}, u_{i} \geq v_{i}, u_{1}^{\prime} \geq v_{1}^{\prime}$,

$f\left(t, u_{1}, u_{2}, u_{1}^{\prime}, T u_{1}, S u_{1}\right)-f\left(t, v_{1}, v_{2}, v_{1}^{\prime}, T v_{1}, S v_{1}\right)$ $\geq-M\left(u_{1}-v_{1}\right)-K\left(u_{2}-v_{2}\right)-N\left(u_{1}^{\prime}-v_{1}^{\prime}\right)-L T\left(u_{1}-v_{1}\right)$,

(ii) Whenever $t \in J$ and $u_{i}, v_{i}(i=1,2) \in Q$ $\equiv\left\{\omega \in C^{1}[J, E] \mid \omega \leq u_{0}, \omega^{\prime} \leq u_{0}^{\prime}\right\}, u_{i} \geq v_{i}, u_{1}^{\prime} \geq v_{1}^{\prime}$,

$f\left(t, u_{1}, u_{2}, u_{1}^{\prime}, T u_{1}, S u_{1}\right)-f\left(t, v_{1}, v_{2}, v_{1}^{\prime}, T v_{1}, S v_{1}\right)$ $\geq-M\left(u_{1}-v_{1}\right)-K\left(u_{2}-v_{2}\right)-N\left(u_{1}^{\prime}-v_{1}^{\prime}\right)-L T\left(u_{1}-v_{1}\right)$,

where $M, K, N, L$ are non-negative constants and satisfy (i) or (ii) in Lemma 1.

$\left(H_{3}\right)$ : (i) There exists $h(t) \in C[J, E]$, for any $u \in G$ and $t \in J$, satisfying $F u(t) \leq h(t)$.

(ii) There exists $g(t) \in C[J, E]$, for any $u \in Q$ and $t \in J$, satisfying $F u(t) \geq g(t)$.

$\left(H_{4}\right)$ : For any countable bounded equicontinuous set $B=\left\{u_{n}\right\} \subset C[J, E]$ and $t \in J$,

$$
\begin{aligned}
& \alpha\left(f(t, B(t)), B(\beta(t)), B^{\prime}(t),(T B)(t),(S B)(t)\right) \\
& \leq c_{1} \alpha(B(t))+c_{2} \alpha(B(\beta(t)))+c_{3} \alpha\left(B^{\prime}(t)\right) \\
& +c_{4} \alpha((T B)(t))+c_{5} \alpha((S B)(t)) .
\end{aligned}
$$

where $c_{i}(i=1,2, \ldots, 5)$ are non-negative constants satisfying one of the following two conditions:

(i) $a c_{5} h_{0}\left(e^{2 a(a+1)\left[c_{1}+c_{2}+c_{3}+2(M+K+N)+2 a L k_{0}+a c_{4} k_{0}\right]}-1\right)$

$$
<\sum_{i=1}^{3} c_{i}+2(M+K+N)+2 a L k_{0}+a c_{4} k_{0},
$$

(ii) $a(a+1)\left[\begin{array}{l}2 \sum_{i=1}^{3} c_{i}+4(M+K+N) \\ +2 a L k_{0}+a c_{4} k_{0}+a c_{5} h_{0}\end{array}\right]<1$.

Theorem 1. Let $P \subset E$ be a normal cone and $\beta(t) \leq t$ on $J$. Assume that conditions $\left(H_{1}\right)(i),\left(H_{2}\right)(i),\left(H_{3}\right)(i)$ and $\left(H_{4}\right)$ hold, then $\operatorname{IVP}(1.1)$ has a minimal solution $u^{*}$ in $G$. Moreover, there exist monotone increasing iterative sequence $\left\{u_{n}\right\} \subset G$ such that $u_{n} \rightarrow u^{*}(n \rightarrow \infty)$ uniformly on $t \in J$, where $u_{n}(t)$ satisfying

$$
\begin{aligned}
& u_{n}(t)=x_{0}+t x_{1} \\
& +\int_{0}^{t}(t-s)\left[\begin{array}{l}
f\left(\begin{array}{l}
s, u_{n-1}(s), u_{n-1}(\beta(s)) \\
u_{n-1}^{\prime}(s), T u_{n-1}(s), S u_{n-1}(s)
\end{array}\right) \\
-M\left(u_{n}-u_{n-1}\right)(s) \\
-K\left(u_{n}-u_{n-1}\right)(\beta(s)) \\
-N\left(u_{n}^{\prime}-u_{n-1}^{\prime}\right)(s)-L T\left(u_{n}-u_{n-1}\right)(s)
\end{array}\right] d s,
\end{aligned}
$$$$
n=1,2, \cdots \text {. }
$$

Proof. First, for any $u_{n-1} \in C^{1}[J, E]$, it is easy to prove that (3.1) has a unique solution $u_{n} \in C[J, E]$.

Next, by(3.1), we have

$$
\begin{aligned}
& u_{n}^{\prime}(t)=x_{1}+\int_{0}^{t}\left[\begin{array}{l}
f\left(\begin{array}{l}
s, u_{n-1}(s), u_{n-1}(\beta(s)), \\
u_{n-1}^{\prime}(s), T u_{n-1}(s), S u_{n-1}(s)
\end{array}\right) \\
-M\left(u_{n}-u_{n-1}\right)(s) \\
-N\left(u_{n}-u_{n-1}\right)(\beta(s)) \\
-N\left(u_{n}^{\prime}-u_{n-1}^{\prime}\right)(s) \\
-L T\left(u_{n}-u_{n-1}\right)(s)
\end{array}\right] d s, \\
& u_{n}(0)=x_{0}, \quad n=1,2, \cdots, \\
& u_{n}^{\prime}(t)=f\left(\begin{array}{l}
t, u_{n-1}(t), u_{n-1}(\beta(t)), \\
u_{n-1}^{\prime}(t), T u_{n-1}(t), S u_{n-1}(t)
\end{array}\right) \\
& -M\left(u_{n}-u_{n-1}\right)(t)-K\left(u_{n}-u_{n-1}\right)(\beta(t))(3.3) \\
& -N\left(u_{n}^{\prime}-u_{n-1}^{\prime}\right)(t)-L T\left(u_{n}-u_{n-1}\right)(t) \text {, } \\
& u_{n}^{\prime}(0)=x_{1}, n=1,2, \cdots \text {. }
\end{aligned}
$$

By (3.3) and $\left(H_{1}\right)(i)$, we have 


$$
\left\{\begin{array}{l}
\left(u_{1}-u_{0}\right)^{\prime \prime}(t) \geq-M\left(u_{1}-u_{0}\right)(t)-K\left(u_{1}-u_{0}\right)(\beta(t)) \\
-N\left(u_{1}^{\prime}-u_{0}^{\prime}\right)(t)-L T\left(u_{1}-u_{0}\right)(t), \\
\left(u_{1}-u_{0}\right)(0)=u_{1}(0)-u_{0}(0)=\theta, \\
\left(u_{1}-u_{0}\right)^{\prime}(0)=u_{1}^{\prime}(0)-u_{0}^{\prime}(0) \geq \theta,
\end{array}\right.
$$

and by Lemma 1 , we can obtain $\left(u_{1}-u_{0}\right)(t) \geq \theta$, $\left(u_{1}-u_{0}\right)^{\prime}(t) \geq \theta, \quad \forall t \in J$. That is $u_{0} \leq u_{1}, u_{0}^{\prime} \leq u_{1}^{\prime}$.

Suppose $u_{k-1}, u_{k} \in G, \quad u_{k-1} \leq u_{k}, \quad u_{k-1}^{\prime} \leq u_{k}^{\prime}$, by (3.3) and $\left(H_{2}\right)(i)$, we have

$$
\left\{\begin{array}{c}
\left(u_{k+1}-u_{k}\right)^{\prime \prime}(t) \geq-M\left(u_{k+1}-u_{k}\right)(t) \\
-K\left(u_{k+1}-u_{k}\right)(\beta(t)) \\
-N\left(u_{k+1}^{\prime}-u_{k}^{\prime}\right)(t)-L T\left(u_{k+1}-u_{k}\right)(t), \\
\left(u_{k+1}-u_{k}\right)(0)=\theta,\left(u_{k+1}-u_{k}\right)^{\prime}(0)=\theta
\end{array}\right.
$$

and so, by Lemma 1 , we have $\left(u_{k+1}-u_{k}\right)(t) \geq \theta$, $\left(u_{k+1}-u_{k}\right)^{\prime}(0) \geq \theta, \quad \forall t \in J$. That is $u_{k} \leq u_{k+1}$, and $u_{k}^{\prime} \leq u_{k+1}^{\prime} \quad u_{k+1} \in G$.

From the above, by induction, it is not difficult to prove that

$$
\begin{aligned}
& u_{0} \leq u_{1} \leq u_{2} \leq \cdots \leq u_{n} \leq \cdots, \\
& u_{0}^{\prime} \leq u_{1}^{\prime} \leq u_{2}^{\prime} \leq \cdots \leq u_{n}^{\prime} \leq \cdots .
\end{aligned}
$$

By (3.1), (3.4) and $\left(H_{3}\right)(i)$, we know

$$
\begin{gathered}
u_{0}(t) \leq u_{n}(t) \leq x_{0}+t x_{1}+\int_{0}^{t}(t-s) h(s) d s \equiv v_{0}(t), \\
\forall t \in J,
\end{gathered}
$$

and so, by (3.2), (3.5) and (3.6), we have

$$
u_{0}^{\prime}(t) \leq u_{n}^{\prime}(t) \leq x_{1}+\int_{0}^{t} h(s) d s, \forall t \in J .
$$

Then, let $B=\left\{u_{n}: n \in N\right\}, \quad B^{\prime}=\left\{u_{n}^{\prime}: n \in N\right\}$, by the normality of $P$ and (3.6) (3.7), we know that $\left\{u_{n}\right\},\left\{u_{n}^{\prime}\right\}$ are bounded sequences in $C[J, E]$.

For any $u_{n-1} \in G$, by $\left(H_{2}\right)(i)$ and $\left(H_{3}\right)(i)$, it is easy to know that

$$
f\left(t, u_{n-1}(t), u_{n-1}(\beta(t)), u_{n-1}^{\prime}(t), T u_{n-1}(t), S u_{n-1}(t)\right)
$$

is bounded. At the same, by (3.2) and (3.3), it is not difficult to show that $\left\{u_{n}\right\},\left\{u_{n}^{\prime}\right\}$ are equicontinuous on $t \in J$.

Let

$$
m(t)=\alpha(B(t)), n(t)=\alpha\left(B^{\prime}(t)\right), \forall t \in J,
$$

and by the uniform boundedness of $B(s)$ and uniform continuity of $k(t, s), h(t, s)$, it is easy to show that $(T B)(s), \quad(S B)(s)$ are bounded and equicontinuous. Therefore, by Lemma 3, we have
$\alpha((T B)(s))=\alpha\left(\left\{\int_{0}^{s} k(s, r) B(r) d r\right\}\right) \leq k_{0} \int_{0}^{s} m(r) d r$,
$\alpha((S B)(s))=\alpha\left(\left\{\int_{0}^{a} h(s, r) B(r) d r\right\}\right) \leq h_{0} \int_{0}^{s} m(r) d r$, then, from (3.1), (3.2), (3.8), (3.9), $\left(H_{4}\right)$, Lemma 2 and Lemma 3, we know $m(t), n(t) \in C\left[J, R^{+}\right]$, and

$$
\begin{aligned}
m(t)= & \alpha(B(t)) \\
\leq & \left.2 a \int_{0}^{t} \alpha\left[\begin{array}{l}
\left.f\left(\begin{array}{l}
s, B(s), B(\beta(s)), \\
B^{\prime}(s), T B(s), S B(s)
\end{array}\right)\right] \\
+2 M B(s)+2 K B(\beta(s)) \\
+2 N B^{\prime}(s)+2 L T B(s)
\end{array}\right]\right) d s \\
\leq & 2 a \int_{0}^{t}\left[\begin{array}{l}
\left(c_{1}+c_{2}\right) \alpha(B(s))+c_{3} \alpha\left(B^{\prime}(s)\right) \\
+c_{4} \alpha((T B)(s))+c_{5} \alpha((S B)(s))
\end{array}\right] d s \\
& +4 a(M+K) \int_{0}^{t} \alpha B(s) d s \\
& +4 a N \int_{0}^{t} \alpha\left(B^{\prime}(s)\right) d s \\
& +4 a L \int_{0}^{t} \alpha((T B)(s)) d s \\
\leq & 2 a\left(c_{1}+c_{2}\right) \int_{0}^{t} m(s) d s+2 a c_{3} \int_{0}^{t} n(s) d s \\
& +\left(2 a c_{4} k_{0}+4 a L k_{0}\right) t \int_{0}^{t} m(s) d s \\
& +2 a c_{4} k_{0} t \int_{0}^{t} m(s) d s+2 a c_{5} h_{0} t \int_{0}^{t} m(s) d s \\
& +4 a\left(M+K \int_{0}^{t} m(s) d s .\right. \\
& +4 a L k_{0} t \int_{0}^{t} m(s) d s \\
& 2 a\left(c_{1}+c_{2}+2 M+2 K\right) \int_{0}^{t} m(s) d s \\
& +\left(2 a c_{0}+4 a N \int_{0}^{t} n(s) d s\right.
\end{aligned}
$$

Similarly, we have

$$
\begin{aligned}
n(t)= & \alpha\left(B^{\prime}(t)\right) \\
\leq & 2\left(c_{1}+c_{2}+2 M+2 K\right) \int_{0}^{t} m(s) d s \\
& +\left(2 c_{3}+4 N\right) \int_{0}^{t} n(s) d s \\
& +\left(2 c_{4} k_{0}+4 L k_{0}\right) t \int_{0}^{t} m(s) d s \\
& +2 c_{5} h_{0} t \int_{0}^{t} m(s) d s .
\end{aligned}
$$

Let $r(t)=\max \{m(t), n(t)\}$, by (3.10), (3.11), we can get

$$
r(t) \leq M_{1} \int_{0}^{t} r(s) d s+M_{2} \int_{0}^{t} r(s) d s+M_{3} \int_{0}^{a} r(s) d s, \forall t \in J,
$$


where $M_{1}=2(a+1)\left[\sum_{i+1}^{3} c_{i}+2(M+K+N)\right]$,

$$
M_{2}=2(a+1)\left(c_{4}+2 L\right) k_{0}, M_{3}=2(a+1) c_{0} h_{0} .
$$

Therefore, by Lemma 4 and the condition (i) or (ii) in $\left(H_{4}\right)$, we see $r(t)=0$. And so $m(t)=0, n(t)=0, \forall t \in J$. Hence $\alpha(B)=0, \alpha\left(B^{\prime}\right)=0$. Then $B, B^{\prime}$ are relatively compact sets in $C[J, E]$. According to (3.4), (3.5) and the normality of $P$, we know $\left\{u_{n}\right\},\left\{u_{n}^{\prime}\right\}$ are convergent sequences respectively in $C[J, E]$. Hence, there exists a $u^{*} \in C[J, E]$ that satisfies $u_{n} \rightarrow u^{*}, \quad u_{n}^{\prime} \rightarrow\left(u^{*}\right)^{\prime}$, $n \rightarrow \infty$. By taking limit in (3.1) as $n \rightarrow \infty$, we have

$u^{*}=x_{0}+t x_{1}+\int_{0}^{t}(t-s) f\left(\begin{array}{l}s, u^{*}(s), u^{*}(\beta(s)), \\ \left(u^{*}\right)^{\prime}(s), T u^{*}(s), \\ S u^{*}(s)\end{array}\right) d s, \forall t \in J$,

so, $u^{*}$ is a solution of (IVP)(1.1) in $G$.

If there exist a $v^{*} \in G$ and $v^{*}$ is also a solution of (IVP)(1.1) in $G$, then $v^{*} \geq u_{0},\left(u^{*}\right)^{\prime} \geq u_{0}^{\prime}$ and

$$
\begin{aligned}
& \left(v^{*}\right)^{\prime \prime}=f\left(\begin{array}{l}
t, v^{*}(t), v^{*}(\beta(t)), \\
\left(v^{*}\right)^{\prime}(t), T v^{*}(t), S v^{*}(t)
\end{array}\right), \\
& v^{*}(0)=x_{0},\left(v^{*}\right)^{\prime}(0)=x_{1} .
\end{aligned}
$$

By (3.3), (3.12) and $\left(H_{2}\right)(i)$, using induction, we can safely obtain

$$
u_{n} \leq v^{*}, u_{n}^{\prime} \leq\left(v^{*}\right)^{\prime}, n=1,2, \cdots .
$$

Letting $n \rightarrow \infty$ in (3.13) and using the normality of $P$, we have $u^{*} \leq v^{*},\left(u^{*}\right)^{\prime} \leq\left(v^{*}\right)^{\prime}$. That is, $u^{*}$ is a minimal solution of (IVP)(1.1) in $G$.

The proof of the theorem is complete.

Theorem 2. Let $P \subset E$ be a normal cone and $\beta(t) \leq t$ on $J$. Assume that conditions (H1)(ii), ( $\left.\mathrm{H}_{2}\right)(\mathrm{ii}),(\mathrm{H} 3)(\mathrm{ii})$ and $(H 4)$ hold, then IVP(1.1) has a maximal solution $v^{*}$ in $Q$. Moreover, there exist monotone decreasing iterative sequence $\left\{v_{n}\right\} \subset Q$ such that $v_{n} \rightarrow v^{*} \quad(n \rightarrow \infty)$ uniformly on $t \in J$, where $v_{n}(t)$ satisfying

$$
\begin{aligned}
& v_{n}(t)=x_{0}+t x_{1} \\
& +\int_{0}^{t}(t-s)\left[\begin{array}{l}
f\left(\begin{array}{l}
s, v_{n-1}(s), v_{n-1}(\beta(s)), \\
v_{n-1}^{\prime}(s), T v_{n-1}(s), S v_{n-1}(s)
\end{array}\right) \\
-M\left(v_{n}-v_{n-1}\right)(s) \\
-K\left(v_{n}-v_{n-1}\right)(\beta(s)) \\
-N\left(v_{n}^{\prime}-v_{n-1}^{\prime}\right)(s)-L T\left(v_{n}-v_{n-1}\right)(s) \\
n=1,2, \cdots
\end{array}\right] d s,(3.14)
\end{aligned}
$$

Proof. The proof of Theorems 2 is almost the same as that of Theorem 1, so we omit it.

Theorem 3. Let $P \subset E$ be a regular cone and $\beta(t) \leq t$ on $J$. Assume that conditions $(H 1)(i),(H 2)(i)$ and $(H 3)(i)$ hold, then the results in Theorem 1 hold.

Proof. According to the proof of Theorems 1, we have (3.4), (3.5), by the regularity of $P$, we can obtain that $u_{n} \leq v^{*}, \quad u_{n}^{\prime} \leq\left(v^{*}\right)^{\prime}, \quad(n \rightarrow \infty)$ uniformly on $t \in J$, the rest of the proof is similar to the proof of Theorems 1 .

Theorem 4. Let $P \subset E$ be a regular cone and $\beta(t) \leq t$ on $J$. Assume that conditions (H1) (ii), (H2) (ii) and (H3)(ii) hold, then the results in Theorem 2 hold.

Proof. By using the similar method of the proof of Theorems 3, we can get the corresponding conclusion.

Remark 1. In (IVP)(1.1), if $f$ does not contain the delayed argument $u(\beta(t))$ and the differential argument $u^{\prime}(t)=u(t)$, then Theorem 1 implies the main results of $[2,6]$, but the conditions in this paper are more extensive than those of $[2,6]$. So the results presented in this paper generalize and unify the results of $[2,6]$.

Remark 2. In paper [1], the author discussed the problem (IVP)(1.1) in which $f$ does not contain $u(\beta(t)), u^{\prime}(t)$ and assumes the increase of $T u$. Obviously in this paper, in the general case, we consider the second-order integrodifferential equation in which $f$ contains $u(\beta(t)), u^{\prime}(t)$ and weaken the increase of $u(t), u^{\prime}(t), T u(t), S u(t)$ and we obtain the minimal and maximal solutions and the iteration sequence of (IVP) (1.1). Moreover, the conditions $(\mathrm{H} 4)$ in this paper are more extensive than those in [1]. Therefore Theorem 1 improves and generalizes the results in [1].

Remark 3. We can see that Theorem 1 is suitable for any measure of non-compactness which is equal to the Kuratowski measure of non-compactness from the proof of Theorem 1.

\section{Acknowledgment}

The authors thanks the referee for hisไher careful reading of the manuscript and useful suggestions.

\section{Support}

This work is supported by the NNSF of China (No.11501342) and the Scientific and Technological Innovation Programs of Higher Education Institutions in Shanxi (No.2014135).

\section{References}

[1] D. J. Guo, Initial value problems for second-order integrodifferential equations in Banach spaces, Nonlinear Analysis, 37(1999): 289-300.

[2] Lishan Liu, Iterative method for solutions and coupled quasisolutions of nonlinear integro-differential equations of mixed type in Banach spaces, Nonlinear Analysis, 42(2000): 583-598. 
[3] D. J. Guo, V. Lakshmikantham, X. Z. Liu, Nonlinear integral equations in abstract spaces, Kluwer Academic Publishers, Dordrecht, 1996.

[4] Hua Su, Lishan Liu, Xiaoyan Zhang, Yonghong Wu, Global solutions of initial value problems for nonlinear second-order integro-differential equations of mixed type in Banach spaces, J.Math>Appl., 330(2007): 1139-1151.

[5] Fangqi Chen, Yushu Chen, On monotone iterative-method for initial value problems of nonlinear second-order integrodifferential equations in Banach space, Appl. Math. Mech., 21(5)(2000): 459-467.

[6] Lishan Liu, The solutions of nonlinear integro-differential equations of mixed type in Banach space, Acta. Math. Sinica, 38(6)(1995): 721-731 (in Chinese).

[7] S. W. Du, V. Lakshmikantham, Monotone iterative technique for differential equations in Banach spaces, J. Math. Anal. Appl., 87(1982): 454-459.

[8] D. J. Guo, V. Lakshmikantham, Nonlinear problems in abstract cones, Academic Press,Boston and New York, 1988.

[9] Dajun Guo, Nonlinear Functional Analysis, 2nd edtion, Science and Technology, Jinan, 2000.

[10] R.P. Agarwal, D. ORegan, P.J.Y. Wong, Positive Solutions of Differential, Difference and Integral Equations, Kluwer Academic Publishers, Dordrecht, 1999.
[11] K.Deimling, Nonlinear Functional Analysis, Springer-Verlag, Berlin, 1985.

[12] T.A. Burton, Differential inequalities for integral and delay differential equations, in: Xinzhi Liu, David Siegel (Eds.), Comparison Methods and Stability Theory, in: Lecture Notes in Pure and Appl. Math., Dekker, New York, 1994.

[13] G. Wang, L. Zhang, G. Song, Integral boundary value problems for first order integro-differential equations with deviating arguments, J. Comput. Appl. Math., 225 (2009) 602-611.

[14] G. Wang, Boundary value problems for systems of nonlinear integro-differential equations with deviating arguments, J. Comput Appl. Math., 234 (2010) 1356-1363.

[15] G. Wang, L. Zhang, G. Song, Systems of first order impulsive functional differential equations with deviating arguments and nonlinear boundary conditions, Nonlinear Analysis,74 (2011) 974982.

[16] G. Wang, Monotone iterative technique for boundary value problems of a nonlinear fractional differential equation with deviating arguments, J. Comput. Appl. Math., 236 (2012) 24252430.

[17] G. Wang, S.K. Ntouyas, L. Zhang, Existence of multiple positive solutions of a nonlinear arbitrary order boundary value problem with advanced arguments, Electronic Journal of Qualitative Theory of Differential Equations 15 (2012) 1-13. 